\title{
MicroRNA-34a inhibits the proliferation and promotes the chemosensitivity of retinoblastoma cells by downregulating Notch1 expression
}

\author{
WEIWEI YIN, FEIFEI GAO and SUOTAO ZHANG \\ Department of Ophthalmology, Laiyang Central Hospital, Yantai, Shandong 265200, P.R. China
}

Received May 21, 2019; Accepted February 11, 2020

DOI: $10.3892 / \mathrm{mmr} .2020 .11238$

\begin{abstract}
MicroRNAs (miRs) are potential therapeutic targets for tumors. The aims of the present study were to investigate the regulatory effects of miR-34a on the proliferation, apoptosis and chemosensitivity of retinoblastoma (RB) cells, and to identify the possible underlying mechanism involving Notch1. It was found that miR-34a was downregulated, and Notch1 was upregulated in HXO-RB44 and Y79 cells. In addition, Notch1 was identified to be a target gene of miR-34a, which could be downregulated by the increased expression of miR-34a. It was demonstrated that miR-34a upregulation and Notch1 downregulation significantly inhibited proliferation, promoted apoptosis and enhanced the carboplatin sensitivity of HXO-RB44 and Y79 cells. The transfection of miR-34a mimics + Notch1 siRNA further enhanced the above anti-tumor responses in HXO-RB44 and Y79 cells. Collectively, the present results suggested that miR-34a may negatively regulate Notch1 expression and may be a potential therapeutic target for RB.
\end{abstract}

\section{Introduction}

Retinoblastoma (RB) is the most common intraocular malignant tumor in pediatric patients $<5$ years old $(1,2)$. Furthermore, $\mathrm{RB}$ has an incidence rate of 1 in 15,000-20,000 live births (3). Currently, the primary approaches for the clinical treatment of RB include laser photo-coagulation, chemotherapy and radiotherapy (4-6). Despite substantial improvements in the treatment of RB, the survival of patients remains poor (7). Thus, it is important to investigate novel therapeutic targets to facilitate the development of treatments for RB.

Previous studies have shown that microRNAs (miRNAs/ miRs) are potential therapeutic targets for tumors (8-11). miRNAs are small endogenous non-coding RNAs,

Correspondence to: Dr Suotao Zhang, Department of Ophthalmology, Laiyang Central Hospital, 111 Changshan Road, Yantai, Shandong 265200, P.R. China

E-mail: zhangsuotao125@163.com

Key words: retinoblastoma, microRNA-34a, Notch1, proliferation, chemosensitivity
18-22 nucleotides in length, that can modulate mRNA translation via binding to the 3'-untranslated region (3'-UTR) of their target genes (12). A variety of miRNAs have been reported to serve oncogenic or anti-tumor roles in $\mathrm{RB}$ cells by regulating cell proliferation, apoptosis, migration, invasion and the cell cycle (9-11). miR-106b promotes the proliferation, migration and invasion of RB cells by inhibiting Zinc Finger and BTB Domain Containing 4 (13). In addition, miR-101-3p suppresses the proliferation of RB cells by targeting Enhancer of zeste homolog 2 and Histone deacetylase 9 (14). Exogenous miR-34a inhibits cell proliferation and increases the apoptotic activity of RB cells (15). However, the specific regulatory role of miR-34a on the chemosensitivity of RB cells is not fully understood.

The anti-tumor potential of miR-34a is closely associated with the regulation of various signaling pathways, such as the Wnt/ $\beta$-Catenin (16), PI3K/AKT/survivin (17) and Notch signaling pathways (18). The Notch signaling pathway is a highly conserved pathway that is involved in the regulation of several fundamental cellular processes, such as cell proliferation, stem cell maintenance and differentiation (19). Furthermore, the Notch family of proteins, which consists of Notch1, Notch2 and Notch3, serve a key regulatory role in RB (20). Li et al (21) indicated that miR-433 suppresses cell proliferation and metastasis in RB by inhibiting Notch1. However, whether the regulatory effect of miR-34a on RB is related with the Notch signaling pathway is unknown.

The present study investigated the regulatory effects of miR-34a on the proliferation and chemosensitivity of RB cells, as well as the related regulatory mechanism involving the Notch signaling pathway. It was found that miR-34a may inhibit the proliferation, and promote the apoptosis and chemosensitivity of RB cells by downregulating Notch1. Therefore, the present study may provide a novel theoretical basis for the treatment of RB.

\section{Materials and methods}

Cell culture. The immortalized human normal retinal vascular endothelial cell line ACBRI-181, and the RB cell lines HXO-RB44 and Y79 were obtained from the American Type Culture Collection. Cells were grown in RPMI-1640 (Gibco; Thermo Fisher Scientific, Inc.) supplemented with $10 \%$ FBS (BD Biosciences) and 1\% penicillin/streptomycin (Gibco; 
Thermo Fisher Scientific, Inc.). All cells were maintained in a humidified $5 \% \mathrm{CO}_{2}$ atmosphere at $37^{\circ} \mathrm{C}$.

Dual-luciferase reporter gene assay. TargetScan software (22) was used to predict the targeting relationship between miR-34a and Notch1. The 3'-UTR containing the miR-34a binding site of Notch1 was amplified and cloned into Psi-CHECK2 reporter vector (Promega Corporation) to construct wild-type (WT) Psi-CHECK2-WT-Notch1-3'-UTR (Notch1-WT) and mutant (MUT) Psi-CHECK2-MUT-Notch1-3'-UTR (Notch1-MUT). For the luciferase assay, miR-34a mimics or miR-34a negative control (NC) mimics $(20 \mathrm{nmol} / \mathrm{l})$ were co-transfected with reporter plasmids $(20 \mathrm{nmol} / \mathrm{l})$ into $\mathrm{HXO}-\mathrm{RB} 44$ and Y79 cells using Lipofectamine ${ }^{\circledR} 2000$ (Invitrogen; Thermo Fisher Scientific, Inc.). HXO-RB44 and Y79 cells were grouped as follows: i) MUT + mimics group, transfected with Notch1-MUT and miR-34a mimics; ii) MUT + NC group, transfected with Notch1-MUT and miR-34a NC mimics; iii) WT + mimics group, transfected with Notch1-WT and miR-34a mimics; and iv) WT + NC group, transfected with Notch1-WT and miR-34a NC mimics. Luciferase activity was assessed using a dual-luciferase kit (Promega Corporation) after $48 \mathrm{~h}$ of transfection, and was normalized to Renilla luciferase activity.

Cell transfection and experimental grouping. miR-34a mimics, Notch1 small interfering RNAs (Notch1 siRNA-1 and -2) and their corresponding NC (miR-34a NC and Notch1 siRNA NC) were supplied by Shanghai GenePharma Co., Ltd. The sequences were: miR-34a mimics forward, 5'-UGGCAG UGUCUUAGCUGGUUGU-3' and reverse, 5'-ACAACCAGC UAAGACACUGCCA-3'; miR-34a NC forward, 5'-UCACAA CCUCCUAGAAAGAGUAGA-3' and reverse, 5'-UCUACU CUUUCUAGGAGGUUGUGA-3'; Notch 1 siRNA-1 forward, 5'-CACCAGUUUGAAUGGUCAAdTdT-3' and reverse, 5'-UUGACCAUUCAAACUGGUGTdTd-3'; Notch 1 siRNA-2 forward, 5'-UGGCGGGAAGUGUGAAGCGdTdT-3' and reverse, 5'-CGCUUCACACUUCCCGCCATdTd-3'; Notch 1 siRNA NC forward, 5'-UUCUCCGAACGUGUCACGUTT-3' and reverse, 5'-ACGUGACACGUUCGGAGAATt-3'. These substances $(20 \mathrm{nmol} / \mathrm{l})$ were transfected into HXO-RB44 and Y79 cells using Lipofectamine ${ }^{\circledR} 2000$ reagent (Invitrogen; Thermo Fisher Scientific, Inc.). HXO-RB44 and Y79 cells were randomly divided into five groups: i) BLANK, cells without transfection; ii) miR-NC + si-NC, transfected with miR-34a NC and Notch1 siRNA NC; iii) miR-34a mimics + si-NC, transfected with miR-34a mimics and Notch1 siRNA NC; iv) miR-NC + Notch1 siRNA, transfected with miR-34a NC and Notch1 siRNA-1; and v) miR-34a mimics + Notch1 siRNA, transfected with miR-34a mimics and Notch1 siRNA-1. All cells were cultured for $48 \mathrm{~h}$ at $37^{\circ} \mathrm{C}$ with $5 \% \mathrm{CO}_{2}$. Some HXO-RB44 and Y79 cells ( $1 \times 10^{5}$ cells/well) were further treated with $40 \mu \mathrm{M}$ Z-VAD (Selleckhchem) for 48 at $37^{\circ} \mathrm{C}$ with $5 \% \mathrm{CO}^{2}$. Cells were randomly divided into four groups: i) Z-VAD; ii) Z-VAD + miR-34a mimics; iii) Z-VAD + Notch1 siRNA; and iv) Z-VAD + miR-34a mimics + Notch1 siRNA.

Cell Counting Kit-8 (CCK-8) assay. The viability of HXO-RB44 and Y79 cells was detected using a CCK-8 assay kit (Invitrogen; Thermo Fisher Scientific, Inc.) according to the manufacturer's protocol. Cells were seeded into 96-well plates $\left(2 \times 10^{3}\right.$ cells/well $)$, and cultured for $24,48,72$ or $96 \mathrm{~h}$ at $37^{\circ} \mathrm{C}$ with $5 \% \mathrm{CO}^{2} . \mathrm{CCK}-8$ solution $(10 \mu \mathrm{l})$ was then added to each well and the cells were incubated at $37^{\circ} \mathrm{C}$ for $2 \mathrm{~h}$. The absorbance of each well was measured at $450 \mathrm{~nm}$ using a microplate reader.

5-Bromo-2-deoxyuridine (BrdU) assay. The proliferation of HXO-RB44 and Y79 cells was measured using a BrdU kit (6813; Cell Signaling Technology, Inc.). Cells were seeded into 96-well plates $\left(1 \times 10^{4}\right.$ cells/well) and incubated overnight at $37^{\circ} \mathrm{C}$. Subsequently, cells were labeled with $10 \mu \mathrm{M}$ BrdU solution for $3 \mathrm{~h}$ at $25^{\circ} \mathrm{C}$, denatured with $100 \mu \mathrm{l}$ FixDenta solution for $30 \mathrm{~min}$ at $25^{\circ} \mathrm{C}$ and incubated with peroxidase-conjugated anti-BrdU antibody $(6813 ; 1: 1,000$; Cell Signaling Technology, Inc.) for $1.5 \mathrm{~h}$ at $25^{\circ} \mathrm{C}$. The absorbance of each well was measured at $450 \mathrm{~nm}$ using a microplate reader.

Flow cytometry assay. The apoptotic rate of HXO-RB44 and Y79 cells was examined using an Annexin V-FITC apoptosis detection kit (KGA108-1, Nanjing KeyGen Biotech Co., Ltd.). Cells were washed three times with PBS, suspended in $500 \mu \mathrm{l}$ binding buffer, and incubated with $5 \mu$ l Annexin V-FITC and $5 \mu \mathrm{l}$ PI for $15 \mathrm{~min}$ in the dark at room temperature. Cell apoptosis was detected using a Cytomics FC500 flow cytometer (Beckman Coulter, Inc.), and the data were analyzed by CytoDiff CXP software (version 2.0, https://www.beckmancoulter.com, Beckman Coulter, Inc.) (23).

Reverse transcription-quantitative PCR (RT-qPCR). Total RNA was extracted from ACBRI-181, HXO-RB44 and Y79 cells using TRIzol ${ }^{\circledR}$ (Invitrogen; Thermo Fisher Scientific, Inc.). A total of $500 \mathrm{ng}$ RNA was then RT into cDNA with a Revert Aid First Strand cDNA Synthesis kit at $42^{\circ} \mathrm{C}$ for $45 \mathrm{~min}$ (Thermo Fisher Scientific, Inc.). RT-qPCR was performed on a RT-qPCR instrument (Bio-Rad Laboratories, Inc.) with SYBR green qPCR Master mix (Thermo Fisher Scientific, Inc.). The program included $94^{\circ} \mathrm{C}$ for $3 \mathrm{~min}, 40$ cycles of $94^{\circ} \mathrm{C}$ for $15 \mathrm{sec}$ and $60^{\circ} \mathrm{C}$ for $30 \mathrm{sec}$. Primers used for RT-qPCR were as follows: miR-34a forward, 5'-GCCACTATGTAGCGGGTT TC-3' and reverse, 5'-ACCTGCGCTAAGAACTGAGG-3'; Notch1 forward, 5'-TCAACGCCGTAGATGACCT-3' and reverse, 5'-TCTCCTCCCTGTTGTTCTGC-3'; Notch1 siRNA-1 forward, 5'-CAUGGUAGUCACUAACAUATT-3' and reverse, 5'-UAUGUUAGUGACUACCAUGTT-3'; Notch1 siRNA-2 forward, 5'-GCACGCGGAUUAAUUUGCCA-3' and reverse, 5'-UGCAAAUUAAUCCGCGUGC-3'; si-NC forward, 5'-UUCUCCGAACGUGUCACGUTT-3' and reverse, 5'-ACGUGACACGUUCGGAGAATT-3'; U6 forward, 5'-CTC GCTTCGGCAGCACA-3' and reverse, 5'-ACGCTTCAC GAATTTGCGT-3'; and GADPH forward, 5'-AGCCACATC GCTCAGACA-3' and reverse, 5'-TGGACTCCACGACGT ACT-3'. Relative expression level was calculated by the $2^{-\Delta \Delta C q}$ method (24).

Western blot analysis. Total proteins were extracted from HXO-RB44 and Y79 cells using RIPA lysis buffer (Beyotime Institute of Biotechnology).Protein concentration was measured with a bicinchoninic acid kit (Yeasen Biotechnology Co., Ltd.). 
Protein samples $(30 \mu \mathrm{g})$ were subjected to $10 \%$ SDS-PAGE and then transferred to a nitrocellulose membrane. After being blocked with $5 \%$ skim milk for $30 \mathrm{~min}$ at $37^{\circ} \mathrm{C}$, the membrane was incubated with the following primary antibodies: Notch1 (14-5785-81; 1:1,000; Chemicon International; Thermo Fisher Scientific, Inc.), p16 (101169-T38; 1:1,000; Sino Biological, Inc.), proliferating cell nuclear antigen (PCNA, 101118-T46; 1:1,000, Sino Biological, Inc.), Bcl-2 (ab185002; 1:1,000; Abcam), Bax (2774s; 1:1,000; Cell Signaling Technology, Inc.), matrix metalloproteinase (MMP)-9 (3852s; 1:1,000; Cell Signaling Technology, Inc.), cleaved caspase-3 (9661s; 1:1,000; Cell Signaling Technology, Inc.) and GAPDH (ab8245; 1:1,000; Abcam) at $4^{\circ} \mathrm{C}$ overnight. The membrane was subsequently incubated with peroxidase-labeled secondary antibody (anti-rabbit IgG; ab6721; 1:5,000; Abcam) at room temperature for $1 \mathrm{~h}$. The protein blots were visualized using an enhanced chemiluminescence kit (Invitrogen; Thermo Fisher Scientific, Inc.). The density of western blot bands was analyzed using Quantity One 1-D Analysis software (version 4.6.9, Bio-Rad Laboratories, Inc.).

Evaluation of drug sensitivity. HXO-RB44 and Y79 cells were seeded into 96 -well plates at a density of $4 \times 10^{3} / \mathrm{ml}$. When the cells had adhered, carboplatin (CBP; Sigma-Aldrich; Merck $\mathrm{KGaA}$ ) was added into each well at a concentration of $0,3,6$, 9 or $12 \mu \mathrm{g} / \mathrm{ml}$. Subsequently, the cells were cultured for $48 \mathrm{~h}$ at $37^{\circ} \mathrm{C}$. Then, CCK-8 reaction solution $(10 \mu \mathrm{l})$ was added into each well and the cells were incubated at $37^{\circ} \mathrm{C}$ for $2 \mathrm{~h}$. The absorbance of each well was measured at $450 \mathrm{~nm}$ using a microplate reader. The inhibition rate (IR; \%) was calculated according to the following formula: $\operatorname{IR}(\%)=\left(1-\mathrm{A}_{\text {Medicine }} / \mathrm{A}_{\text {Control }}\right)$ $\mathrm{x} 100 \%$. The CBP concentration that caused the death of half of HXO-RB44 or Y79 cells was calculated, and considered the $\mathrm{IC}_{50}$.

Statistical analysis. All statistical analyses were performed using SPSS version 22.0 (IBM Corp.). All experiments were repeated three times. Data are presented as the mean \pm standard deviation. A one-way ANOVA followed by Tukey's post hoc test was used to analyze the differences among multiple groups. $\mathrm{P}<0.05$ was considered to indicate a statistically significant difference.

\section{Results}

miR-34a is downregulated and Notchl is upregulated in $R B$ cells. The expression levels of miR-34a and Notch1 in ACBRI-181, HXO-RB44 and Y79 cells were detected using RT-qPCR. It was found that the mRNA expression level of miR-34a was significantly lower in HXO-RB44 and Y79 cells compared with ACBRI-181 cells $(\mathrm{P}<0.05$; Fig. 1A). However, the mRNA expression level of Notch1 in HXO-RB44 and Y79 cells was significantly higher compared with ACBRI-181 cells $(\mathrm{P}<0.05$; Fig. 1B).

miR-34a downregulates Notch1 in RB cells. Notch1 was silenced in HXO-RB44 and Y79 cells via the transfection of si-Notch1-1 and si-Notch1-2. The RT-qPCR results indicated that that the expression levels of Notch1 was significantly decreased by si-Notch1-1 and si-Notch1-2 ( $\mathrm{P}<0.001$; Fig. 1C).
In addition, miR-34a was overexpressed in HXO-RB44 and Y79 cells via the transfection of miR-34a mimics. The RT-qPCR results showed that the mRNA expression levels of miR-34a were significantly increased by miR-34a mimics in HXO-RB44 and Y79 cells ( $\mathrm{P}<0.001$; Fig. 1D). Furthermore, the effect of miR-34a mimics on the expression levels of Notch1 was analyzed using si-Notch1-1. Compared with the BLANK group, the mRNA and protein expression levels of Notch1 in HXO-RB44 and Y79 cells were significantly decreased by miR-34a mimics and Notch1 siRNA transfections $(\mathrm{P}<0.05)$. In addition, it was found that Notch1 was further downregulated in the miR-34a mimics + Notch1 siRNA group compared with the miR-NC + Notch 1 siRNA group ( $\mathrm{P}<0.05$; Fig. 1E and F).

Notchl is the target gene of miR-34a. TargetScan analysis predicted that the binding site of Notch1 to miR-34a was at the 3'-UTR region (Fig. 2A). Based on the result that the Notch1 promoter contains the putative miR-34a binding site, the target relationship between Notch1 and miR-34a was further analyzed by dual-luciferase reporter gene assay. It was demonstrated that the luciferase activity of the WT + mimics group was significantly decreased compared with the WT + NC group $(\mathrm{P}<0.05$; Fig. 2B).

miR-34a upregulation and Notch1 downregulation inhibit the proliferation of $R B$ cells. The viability of HXO-RB44 and Y79 cells was detected with a CCK-8 assay. The viability of HXO-RB44 and Y79 cells was significantly decreased by the transfection of miR-34a mimics and Notch1 siRNA compared with the BLANK group $(\mathrm{P}<0.05$; Fig. $3 \mathrm{~A})$. Furthermore, the expression levels of the proliferation-related proteins p16 and PCNA were measured using western blotting. Compared with the BLANK group, PCNA expression levels were significantly decreased, and p16 expression levels were significantly increased by the transfection of miR-34a mimics and Notch1 siRNA in HXO-RB44 and Y79 cells $(\mathrm{P}<0.05$; Fig. 3B). The proliferation of RB cells was further analyzed using a BrdU assay. Compared with the BLANK group, the optical density (OD) 450 values of HXO-RB44 and Y79 cells were significantly decreased by miR-34a mimics and Notch1 siRNA $(\mathrm{P}<0.05$; Fig. 3C). Furthermore, it was found that the transfection of miR-34a mimics + Notch1 siRNA decreased cell viability, upregulated p16 expression, downregulated PCNA expression and decreased OD450 values of HXO-RB44 and Y79 cells, compared with the transfection of miR-34a mimics and Notch1 siRNA alone ( $\mathrm{P}<0.05$; Fig. 3A-C).

miR-34a upregulation and Notchl downregulation promotes the apoptosis of $R B$ cells. The apoptotic rate of HXO-RB44 and Y79 cells was detected using Annexin/PI double staining. It was demonstrated that the transfection of miR-34a mimics and Notch1 siRNA alone significantly increased the percentage of apoptotic cells compared with the BLANK group $(\mathrm{P}<0.05)$. Furthermore, the transfection of miR-34a mimics + Notch1 siRNA further increased the percentage of apoptotic cells $(\mathrm{P}<0.05$; Fig. 4A). The expression levels of the apoptosis-related proteins Bax, Bcl-2, MMP-9 and cleaved caspase- 3 were measured by western blotting. Compared with the BLANK group, the transfection of miR-34a mimics and Notch1 siRNA alone significantly upregulated Bax and 
A

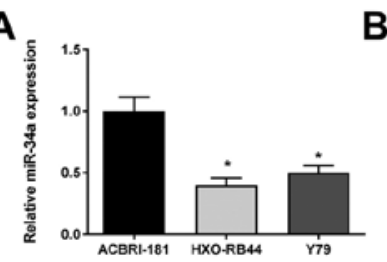

E

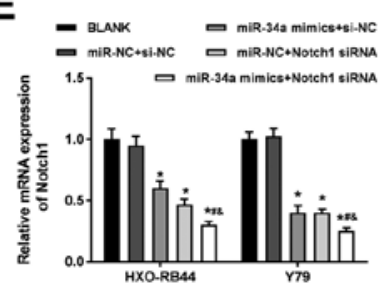

B

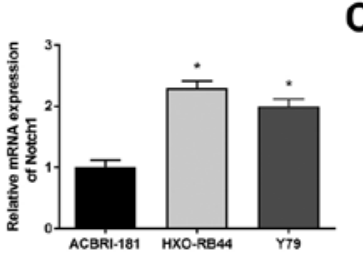

C

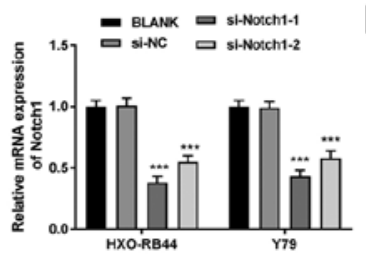

D

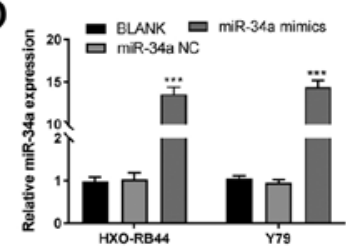

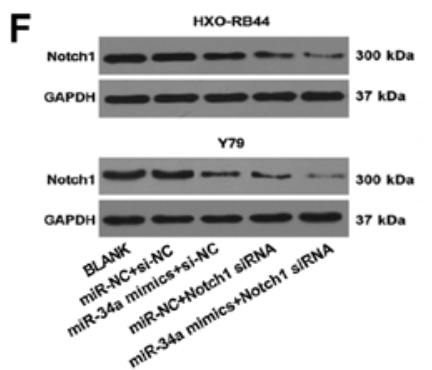

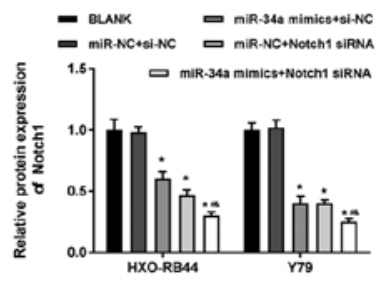

Figure 1. Expression levels of miR-34a and Notch1 in RB cells. (A) mRNA expression levels of miR-34a was detected by RT-qPCR in ACBRI-181, HXO-RB44 and Y79 cells. (B) mRNA expression levels of Notch1 were detected by RT-qPCR in ACBRI-181, HXO-RB44 and Y79 cells. "P<0.05 vs. ACBRI-181 group. (C) mRNA expression of Notch1 was detected by RT-qPCR in si-Notch1 transfected HXO-RB44 and Y79 cells. ${ }^{* * *} \mathrm{P}<0.001$ vs. BLANK and si-NC group. (D) mRNA expression levels of miR-34a in HXO-RB44 and Y79 cells $48 \mathrm{~h}$ after transfection. ${ }^{* * *} \mathrm{P}<0.001$ vs. BLANK and miR-34a NC group. (E) mRNA expression levels of Notch1 in HXO-RB44 and Y79 cells $48 \mathrm{~h}$ post-transfection. (F) Protein expression levels of Notch1 were detected by western blotting in HXO-RB44 and Y79 cells at $48 \mathrm{~h}$ post-transfection. ${ }^{\mathrm{P}} \mathrm{P}<0.05$ vs. BLANK and miR-NC + si-NC group. ${ }^{\text {"P}} \mathrm{P}<0.05$ vs. miR-34a mimics + si-NC group. ${ }^{\text {\& }} \mathrm{P}<0.05$ vs. miR-NC + Notch1 siRNA group. Data are presented as the mean \pm standard deviation, with three replicates. siRNA, small interfering RNA; NC, negative control; miR, microRNA; RT-qPCR, reverse transcription-quantitative PCR.

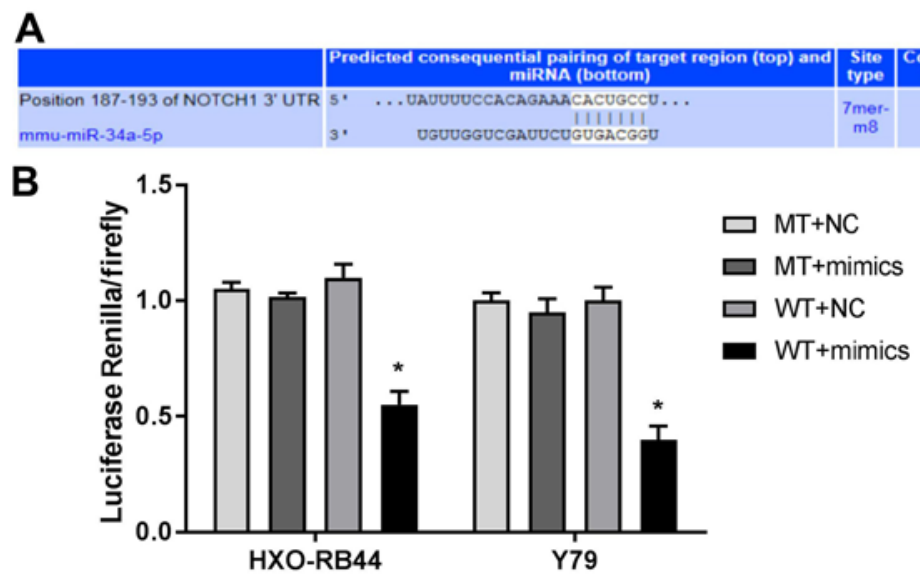

Figure 2. Notch1 is the target gene of miR-34a. (A) Binding target of Notch1 and miR-34a was predicted by TargetScan software. (B) Luciferase activity was measured using a dual-luciferase reporter gene assay. Data are presented as the mean \pm standard deviation, with three replicates. ${ }^{*} \mathrm{P}<0.05 \mathrm{vs}$. WT $+\mathrm{NC}$ group. WT, wild-type; MUT, mutant; NC, negative control; miR, microRNA; 3'-UTR, 3' untranslated region.

cleaved caspase-3 expression, and downregulated Bcl-2 and MMP-9 expression $(\mathrm{P}<0.05)$. Furthermore, it was found that the changes in expression of these apoptosis-related proteins were more significant in cells transfected with miR-34a mimics + Notch1 siRNA, compared with those transfected with miR-34a mimics and Notch 1 siRNA alone $(\mathrm{P}<0.05$; Fig. 4B and C). In addition, the inhibiting effect of Z-VAD, a caspase inhibitor, on cleaved caspase- 3 expression level was significantly reversed by the transfection of miR-34a mimics and Notch1 siRNA alone, and particularly by the transfection of miR-34a mimics + Notch1 siRNA (P<0.05; Fig. 4D).

miR-34a upregulation and Notch1 downregulation increases the sensitivity of RB cells to CBP. The sensitivity of HXO-RB44 and Y79 cells to CBP was evaluated by CKK-8 assay. Compared with the BLANK group, the transfection of miR-34a mimics and Notch1 siRNA alone significantly increased the IR of cells treated with different concentrations of CBP. Furthermore, it was demonstrated that the transfection of miR-34a mimics + Notch1 siRNA further increased the IR ( $\mathrm{P}<0.05$; Fig. 5A). These results suggest that the CBP $\mathrm{IC}_{50}$ was significantly lower in cells transfected with miR-34a mimics and Notch1 siRNA alone compared with the BLANK group. The $\mathrm{CBP} \mathrm{IC}_{50}$ was lowest in cells transfected with miR-34a mimics + Notch1 siRNA compared with the different groups $(\mathrm{P}<0.05$; Fig. 5B).

\section{Discussion}

miRNAs serve key regulatory roles in the occurrence and development of RB $(25,26)$. It has been previously reported 

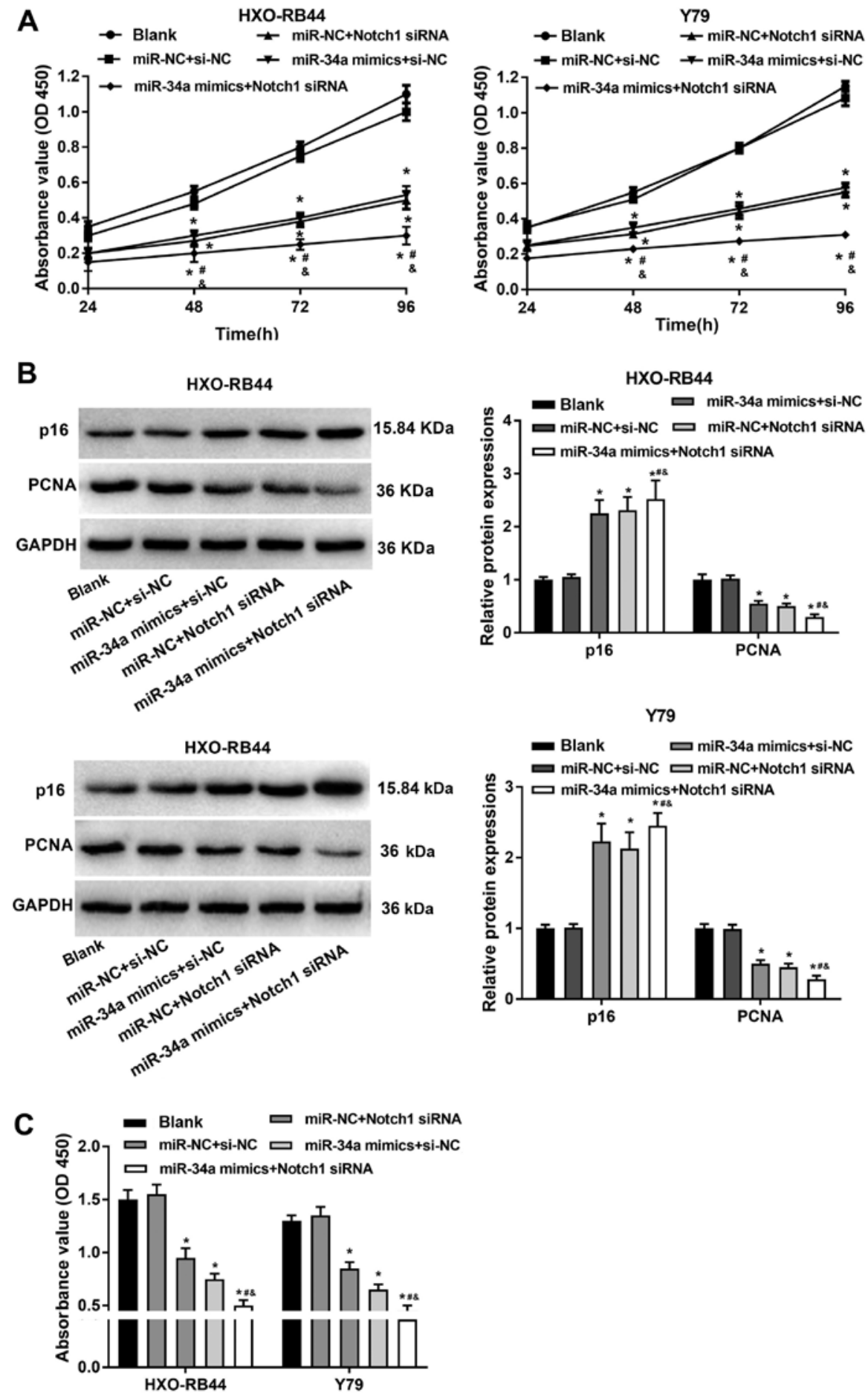

Figure 3. miR-34a inhibits the proliferation of HXO-RB44 and Y79 cells. (A) Cell viability of transfected HXO-RB44 and Y79 cells was measured using a Cell Counting Kit-8 assay. (B) Protein expression levels of p16 and PCNA were measured by western blotting 48 h post-transfection. (C) OD450 values of cells were measured using a 5-Bromo-2-deoxyuridine assay $48 \mathrm{~h}$ post-transfection. Data are presented as the mean \pm standard deviation, with three replicates. ${ }^{\text {} P}<0.05$ vs. BLANK and miR-NC + si-NC group. ${ }^{\text {} P}<0.05$ vs. miR-34a mimics + si-NC group. ${ }^{\circledR} \mathrm{P}<0.05$ vs. miR-NC + Notch1 siRNA group. OD, optical density; siRNA, small interfering RNA; miR, microRNA; NC, negative control; PCNA, proliferating cell nuclear antigen.

that miR-34a expression levels are decreased in breast cancer, cervical cancer, pancreatic cancer, cholangiocarcinoma and RB (15,27-29). In relation to this, the results of the present study showed that the expression levels of miR-34a were significantly lower in RB cell lines compared with human normal retinal vascular endothelial cells. Therefore, the present results suggested that miR-34a is downregulated in RB, which is consistent with the aforementioned previous studies. In addition, previous studies suggested that miR-34a acts as a potential tumor suppressor by modulating cell proliferation, apoptosis, migration and invasion (15). miR-34a suppresses colorectal cancer metastasis by attenuating cell migration and invasion (30). In addition, miR-34a significantly decreases the proliferation rate and increases the apoptotic rate of gastric cancer cells (31). Exogenous miR-34a also inhibits the proliferation and promotes the 
A
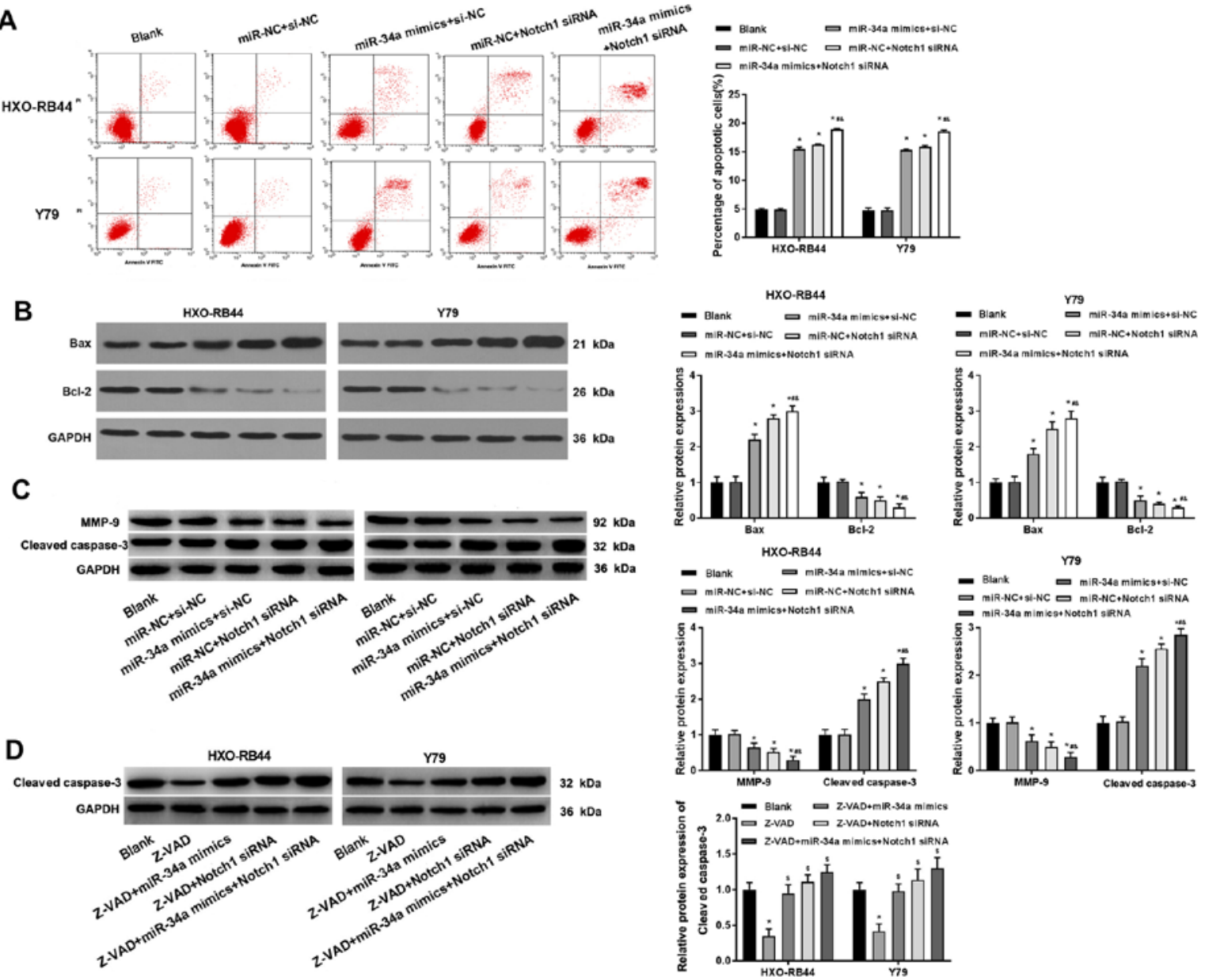

Figure 4. miR-34a promotes the apoptosis of HXO-RB44 and Y79 cells. (A) Cell apoptosis of HXO-RB44 and Y79 cells was detected by flow cytometry $48 \mathrm{~h}$ post-transfection. (B) Protein expression levels of Bax and Bcl-2 were measured by western blotting. (C) Protein expression levels of MMP-9 and cleaved caspase-3. "P $<0.05$ vs. BLANK and miR-NC + si-NC group. (D) Protein expression levels of cleaved caspase-3 were measured by western blot in Z-VAD-treated HXO-RB44 and Y79 cells at $48 \mathrm{~h}$ post-treatment. ${ }^{~} \mathrm{P}<0.05$ vs. BLANK. Data are presented as the mean \pm standard deviation, with three replicates. ${ }^{\prime \prime} \mathrm{P}<0.05$ vs. miR-34a mimics + si-NC group. ${ }^{\circledR} \mathrm{P}<0.05$ vs. miR-NC + Notch1 siRNA group. ${ }^{\$} \mathrm{P}<0.05$ vs. Z-VAD group. siRNA, small interfering RNA; miR, microRNA; NC, negative control; MMP, matrix metalloproteinase.
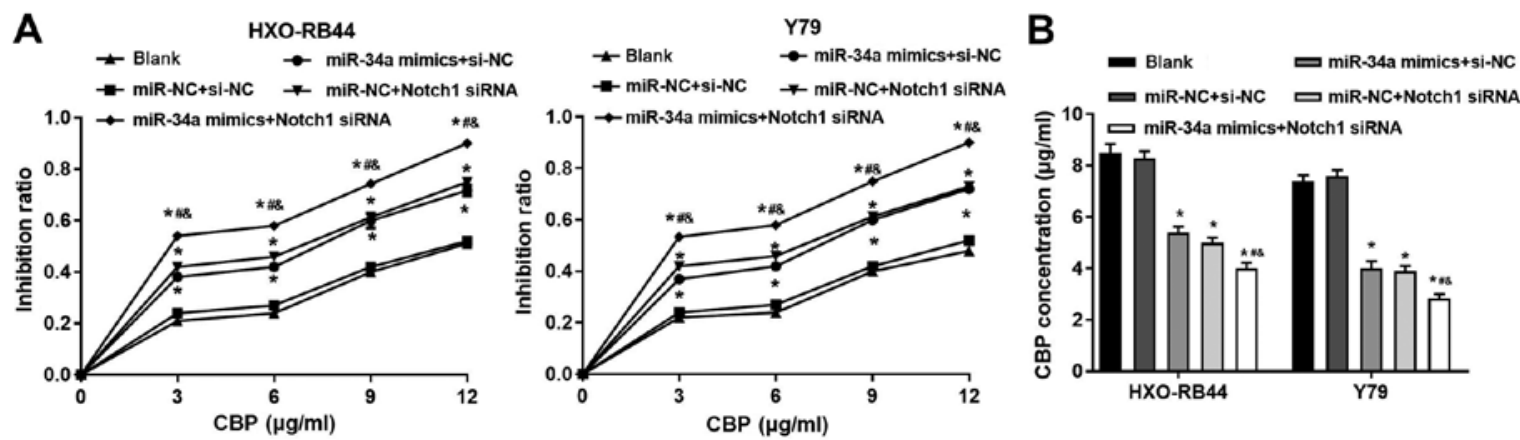

Figure 5. miR-34a promotes CBP sensitivity of HXO-RB44 and Y79 cells. (A) Inhibition ratios of CBP-treated HXO-RB44 and Y79 cells were detected using a Cell Counting Kit-8 assay $48 \mathrm{~h}$ post-treatment. (B) CBP IC $\mathrm{I}_{50}$. Data are presented as the mean \pm standard deviation, with three replicates. "P $<0.05$ vs. BLANK group and miR-NC + si-NC group. ${ }^{\#} \mathrm{P}<0.05$ vs. miR-34a mimics + si-NC group. ${ }^{\circledR} \mathrm{P}<0.05$ vs. miR-NC + Notch1 siRNA group. miR, microRNA; siRNA, small interfering RNA; CBP, carboplatin; NC, negative control.

apoptosis of RB cells (15). In the present study, it was found that miR-34a inhibited the proliferation and promoted the apoptosis of HXO-RB44 and Y79 cells. Therefore, the present results are consistent with previous studies, and demonstrated that miR-34a may be a potential therapeutic target for RB.
The Notch signaling pathway has been identified as a potential therapeutic target for a variety of cancer types, including pancreatic cancer (32), hepatocellular carcinoma (33) and RB (20). In addition, the Notch family of proteins serve vital roles in retinal development (34). During early retinal development in mammals, Notch1 and Notch3 are primarily 
present in the central portion of the retina, and Notch2 is predominantly expressed in the retinal periphery and pigment epithelial cells $(35,36)$. A previous study showed that Notch1 expression levels were significantly higher compared with Notch 2 and Notch3 expression levels in Y79 cells (37). The present results suggested that Notch1 was upregulated in RB cells compared with human normal retinal vascular endothelial cells, consistent with the results from a previous study, which identified that Notch1 is highly expressed in human RB cells (20). Furthermore, previous studies showed that Notch1 is a target of miR-34a (38-40). miR-34a inhibits cell invasion via the downregulation of Notch1 in cervical carcinoma and choriocarcinoma (38). In addition, miR-34a inhibits the proliferation, and induces the apoptosis of glioblastoma cells by targeting Notch1 (39). miR-34a inhibits the proliferation and invasion of endometrial cancer cells by downregulating Notch1 (40). In line with previous studies, the present results indicated that Notch1 may be a target gene of miR-34a, and that Notch1 may be downregulated by miR-34a upregulation. In addition, downregulation of Notch1 inhibited the proliferation and promoted apoptosis of HXO-RB44 and Y79 cells. Thus, Notch1 may act as an oncogene in RB (37). In addition, miR-34a-induced downregulation of Notch1 may contribute to the anti-tumor response. Thus, miR-34a may inhibit the proliferation and promote the apoptosis of RB cells by downregulating Notch1.

Pediatric patients with $\mathrm{RB}$ are primarily treated with chemotherapy using drugs such as CBP, vincristine and etoposide (ETO) (41). However, drug resistance greatly limits the prognosis of patients with RB $(42,43)$. Previous studies have revealed that miR-34a can, to a certain extent, increase the chemosensitivity of RB cells $(44,45)$. miR-34a downregulation elevates the survival rate and viability of RB cells following carboplatin, adriamycin and vincristine treatment (44). Furthermore, miR-34a restores ETO and CBP chemosensitivity, and increases the apoptosis of RB cells (45). The present results suggested that miR-34a increased the IR and CBP $\mathrm{IC}_{50}$ of HXO-RB44 and Y79 cells. This result is consistent with previous studies, and indicates that miR-34a promotes the chemosensitivity of RB cells to CBP treatment. In addition, miR-34a increases the chemosensitivity of breast cancer stem cells to Paclitaxel by downregulating Notch1 (46). miR-34a sensitizes the chemosensitivity of breast cancer cells to adriamycin by targeting Notch1 (47). As Notch1 is a target gene of miR-34a, the present results suggested that miR-34a may improve the chemosensitivity of RB cells by downregulating Notch1, and thus aid in the treatment of RB.

In conclusion, it was found that miR-34a was downregulated and Notch1 was upregulated in HXO-RB44 and Y79 cells. In addition, miR-34a upregulation inhibited the proliferation, promoted the apoptosis and enhanced the CBP chemosensitivity of RB cells by downregulating Notch1. The present results provide a novel regulatory mechanism of miR-34a in RB cells, and may facilitate the treatment of RB as overexpression of miR-34a may be a potential therapeutic strategy.

\section{Acknowledgements}

Not applicable.

\section{Funding}

No funding was received.

\section{Availability of data and materials}

The datasets generated and/or analyzed during the current study are not publicly available due to research related to future studies, but are available from the corresponding author on reasonable request.

\section{Authors' contributions}

SZ was responsible for the conception and design of the study, and development of the manuscript. FG and WY performed the experiments, and were involved in the collection and supervision of data. WY participated in drafting the manuscript or revising it critically for important intellectual content. All authors read and approved the final manuscript.

\section{Ethics approval and consent to participate}

Not applicable.

\section{Patient consent for publication}

Not applicable.

\section{Competing interests}

The authors declare that they have no competing interests.

\section{References}

1. Dimaras H, Dimba EA and Gallie BL: Challenging the global retinoblastoma survival disparity through a collaborative research effort. Br J Ophthalmol 94: 1415-1416, 2010.

2. Mahoney MC, Burnett WS, Majerovics A and Tanenbaum H: The epidemiology of ophthalmic malignancies in New York State. Ophthalmology 97: 1143-1147, 1990.

3. Ortiz MV and Dunkel IJ: Retinoblastoma. J Child Neurol 31: 227-236, 2016

4. Shields CL and Shields JA: Retinoblastoma management: Advances in enucleation, intravenous chemoreduction, and intra-arterial chemotherapy. Curr Opin Ophthalmol 21: 203-212, 2010.

5. Assayag F, Nicolas A, Vacher S, Dehainault C, Bieche I, Meseure D, Aerts I, Cassoux N, Houdayer C, Doz F, et al: Combination of Carboplatin and Bevacizumab Is an Efficient Therapeutic Approach in Retinoblastoma Patient-Derived Xenografts. Invest Ophthalmol Vis Sci 57: 4916-4926, 2016.

6. Shehata HH, Abou Ghalia AH, Elsayed EK, Ahmed Said AM and Mahmoud SS: Clinical significance of high levels of survivin and transforming growth factor beta-1 proteins in aqueous humor and serum of retinoblastoma patients. J AAPOS 20: 444 e1-444 e9, 2016.

7. Kivelä T: The epidemiological challenge of the most frequent eye cancer: Retinoblastoma, an issue of birth and death. Br J Ophthalmol 93: 1129-1131, 2009.

8. de Carvalho IN, de Freitas RM and Vargas FR: Translating microRNAs into biomarkers: What is new for pediatric cancer? Med Oncol 33: 49, 2016.

9. Fabian MR, Sonenberg N and Filipowicz W: Regulation of mRNA translation and stability by microRNAs. Annu Rev Biochem 79: 351-379, 2010.

10. Liu Y,Zhou Y, Gong X and Zhang C: MicroRNA-30a-5p inhibits the proliferation and invasion of gastric cancer cells by targeting insulin-like growth factor 1 receptor. Exp Ther Med 14: 173-180, 2017. 
11. Chandra S, Vimal D, Sharma D, Rai V, Gupta SC and Chowdhuri DK: Role of miRNAs in development and disease: Lessons learnt from small organisms. Life Sci 185: 8-14, 2017.

12. Jin $\mathrm{D}$ and Lee H: Prioritizing cancer-related microRNAs by integrating microRNA and mRNA datasets. Sci Rep 6: 35350, 2016

13. Bu W, Wang $\mathrm{Y}$ and Min X: MicroRNA-106b promotes the proliferation, migration and invasion of retinoblastoma cells by inhibiting the expression of ZBTB4 protein. Exp Ther Med 16: 4537-4545, 2018.

14. Jin Q, He W, Chen L, Yang Y, Shi K and You Z: MicroRNA-101-3p inhibits proliferation in retinoblastoma cells by targeting EZH2 and HDAC9. Exp Ther Med 16: 1663-1670, 2018.

15. Dalgard CL, Gonzalez M, deNiro JE and O'Brien JM: Differential microRNA-34a expression and tumor suppressor function in retinoblastoma cells. Invest Ophthalmol Vis Sci 50: 4542-4551, 2009.

16. Si W, Li Y, Shao H, Hu R, Wang W, Zhang K and Yang Q: MiR-34a Inhibits Breast Cancer Proliferation and Progression by Targeting Wnt 1 in Wnt/ $\beta$-Catenin Signaling Pathway. Am J Med Sci 352: 191-199, 2016.

17. Cao W, Yang W, Fan R, Li H, Jiang J, Geng M, Jin Y and Wu Y: miR-34a regulates cisplatin-induce gastric cancer cell death by modulating PI3K/AKT/survivin pathway. Tumour Biol 35 : $1287-1295,2014$

18. Wang XP, Zhou J, Han M, Chen CB, Zheng YT, He XS and Yuan XP: MicroRNA-34a regulates liver regeneration and the development of liver cancer in rats by targeting Notch signaling pathway. Oncotarget 8: 13264-13276, 2017.

19. Kidd S, Lockett TJ and Young MW: The Notch locus of Drosophila melanogaster. Cell 34: 421-433, 1983.

20. Xiao W, Chen X and He M: Inhibition of the Jagged/Notch pathway inhibits retinoblastoma cell proliferation via suppressing the PI3K/Akt, Src, p38MAPK and Wnt/ $\beta$ catenin signaling pathways. Mol Med Rep 10: 453-458, 2014

21. Li X, Yang L, Shuai T, Piao T and Wang R: MiR-433 inhibits retinoblastoma malignancy by suppressing Notch1 and PAX6 expression. Biomed Pharmacother 82: 247-255, 2016.

22. Nam JW, Rissland OS, Koppstein D, Abreu-Goodger C, Jan CH, Agarwal V, Yildirim MA, Rodriguez A and Bartel DP: Global analyses of the effect of different cellular contexts on microRNA targeting. Mol Cell 53: 1031-1043, 2014.

23. Faucher JL, Lacronique-Gazaille C, Frébet E, Trimoreau F, Donnard M, Bordessoule D, Lacombe F and Feuillard J: "6 markers $/ 5$ colors" extended white blood cell differential by flow cytometry. Cytometry A 71: 934-944, 2007.

24. Livak KJ and Schmittgen TD: Analysis of relative gene expression data using real-time quantitative PCR and the 2(-Delta Delta C(T)) Method. Methods 25: 402-408, 2001

25. Castro-Magdonel BE, Orjuela M, Camacho J, García-Chéquer AJ, Cabrera-Muñoz L, Sadowinski-Pine S, Durán-Figueroa N, Orozco-Romero MJ, Velázquez-Wong AC, Hernández-Ángeles $\mathrm{A}$, et al: miRNome landscape analysis reveals a 30 miRNA core in retinoblastoma. BMC Cancer 17: 458, 2017.

26. Montoya V, Fan H, Bryar PJ, Weinstein JL, Mets MB, Feng G, Martin J, Martin A, Jiang H and Laurie NA: Novel miRNA-31 and miRNA-200a-Mediated Regulation of Retinoblastoma Proliferation. PLoS One 10: e0138366, 2015.

27. Lodygin D, Tarasov V, Epanchintsev A, Berking C, Knyazeva T, Körner H, Knyazev P, Diebold J and Hermeking H: Inactivation of miR-34a by aberrant $\mathrm{CpG}$ methylation in multiple types of cancer. Cell Cycle 7: 2591-2600, 2008.

28. Wang X, Xie Y and Wang J: Overexpression of MicroRNA-34a-5p Inhibits Proliferation and Promotes Apoptosis of Human Cervical Cancer Cells by Downregulation of Bcl-2. Oncol Res 26: 977-985, 2018.

29. Kwon H, Song K, Han C, Zhang J, Lu L, Chen W and Wu T: Epigenetic Silencing of miRNA-34a in Human Cholangiocarcinoma via EZH2 and DNA Methylation: Impact on Regulation of Notch Pathway. Am J Pathol 187: 2288-2299, 2017.
30. Zhang X, Ai F, Li X, Tian L, Wang X, Shen S and Liu F: MicroRNA-34a suppresses colorectal cancer metastasis by regulating Notch signaling. Oncol Lett 14: 2325-2333, 2017.

31. Deng X, Zheng H, Li D, Xue Y, Wang Q, Yan S, Zhu Y and Deng M: MicroRNA-34a regulates proliferation and apoptosis of gastric cancer cells by targeting silent information regulator 1 . Exp Ther Med 15: 3705-3714, 2018.

32. Ponnurangam S, Dandawate PR, Dhar A, Tawfik OW, Parab RR, Mishra PD, Ranadive P, Sharma R, Mahajan G, Umar S, et al: Quinomycin A targets Notch signaling pathway in pancreatic cancer stem cells. Oncotarget 7: 3217-3232, 2016.

33. Ke X, Zhao Y, Lu X, Wang Z, Liu Y, Ren M, Lu G, Zhang D, Sun Z, Xu Z, et al: TQ inhibits hepatocellular carcinoma growth in vitro and in vivo via repression of Notch signaling. Oncotarget 6: 32610-32621, 2015 .

34. Mitter D, Ullmann R, Muradyan A, Klein-Hitpass L, Kanber D, Ounap K, Kaulisch M and Lohmann D: Genotype-phenotype correlations in patients with retinoblastoma and interstitial $13 \mathrm{q}$ deletions. Eur J Hum Genet 19: 947-958, 2011.

35. Lindsell CE, Boulter J, diSibio G, Gossler A and Weinmaster G Expression patterns of Jagged, Delta1, Notch1, Notch2, and Notch3 genes identify ligand-receptor pairs that may function in neural development. Mol Cell Neurosci 8: 14-27, 1996.

36. Bao ZZ and Cepko CL: The expression and function of Notch pathway genes in the developing rat eye. J Neurosci 17: 1425-1434, 1997.

37. Asnaghi L, Tripathy A, Yang Q, Kaur H, Hanaford A, Yu W and Eberhart CG: Targeting Notch signaling as a novel therapy for retinoblastoma. Oncotarget 7: 70028-70044, 2016.

38. Pang RT, Leung CO, Ye TM, Liu W, Chiu PC, Lam KK, Lee KF and Yeung WS: MicroRNA-34a suppresses invasion through downregulation of Notch1 and Jagged1 in cervical carcinoma and choriocarcinoma cells. Carcinogenesis 31: 1037-1044, 2010.

39. Li WB, Ma MW, Dong LJ, Wang F, Chen LX and Li XR: MicroRNA-34a targets notch1 and inhibits cell proliferation in glioblastoma multiforme. Cancer Biol Ther 12: 477-483, 2011.

40. Wang Z, Wang W, Huang K, Wang Y, Li J and Yang X: MicroRNA-34a inhibits cells proliferation and invasion by downregulating Notch1 in endometrial cancer. Oncotarget 8: 111258-111270, 2017.

41. Jo DH, Lee K, Kim JH, Jun HO, Kim Y, Cho YL, Yu YS, Min JK and Kim JH: L1 increases adhesion-mediated proliferation and chemoresistance of retinoblastoma. Oncotarget 8: 15441-15452, 2017.

42. Rao R and Honavar SG: Retinoblastoma. Indian J Pediatr 84: 937-944, 2017.

43. Saakyan SV, Tsygankov AY, Moiseeva NI, Karamysheva AF Zhil'tsova MG and Tadevosyan SS: Retinoblastoma Cell Culturing and Evaluation of Their Drug Resistance. Bull Exp Biol Med 165: 148-153, 2018.

44. Yang G, Fu Y, Lu X, Wang M, Dong H and Li Q: miR 34a regulates the chemosensitivity of retinoblastoma cells via modulation of MAGE A/p53 signaling. Int J Oncol 54: 177-187, 2019.

45. Liu K, Huang J, Xie M, Yu Y, Zhu S, Kang R, Cao L, Tang D and Duan X: MIR34A regulates autophagy and apoptosis by targeting HMGB1 in the retinoblastoma cell. Autophagy 10: 442-452, 2014

46. Kang L, Mao J, Tao Y, Song B, Ma W, Lu Y, Zhao L, Li J, Yang B and Li L: MicroRNA-34a suppresses the breast cancer stem cell-like characteristics by downregulating Notch1 pathway. Cancer Sci 106: 700-708, 2015.

47. Li XJ, Ji MH, Zhong SL, Zha QB, Xu JJ, Zhao JH and Tang JH: MicroRNA-34a modulates chemosensitivity of breast cancer cells to adriamycin by targeting Notch1. Arch Med Res 43: 514-521, 2012. 